\title{
Scrutiny of Brain CT Scan Images by using Corrective Clustering Technique
}

\author{
Ehsan Banihashemi \\ Department of Computer \\ Science, Ferdows Branch, \\ Islamic Azad University, \\ Ferdows, Iran
}

\author{
Meysam Dabiri \\ Moghadam \\ Department of Computer \\ Science, Ferdows Branch, \\ Islamic Azad University, \\ Ferdows, Iran
}

\author{
Hamidreza Ghaffary \\ Department of Computer \\ Science, Ferdows Branch, \\ Islamic Azad University, \\ Ferdows, Iran
}

\begin{abstract}
In the paper we present an approach to Introduce automation of brain CT image analysis Because CT Scan method that used especially for the diagnosis of stroke and can detect bleeding in stroke due to a blocked artery, of course Images from a CT scan resolution is low relatively. Therefore, the grayscale images resolution is scant and makes detection difficult. We can use bioinformatics and artificial coloring techniques by image processing quality added and is more sensitive in outstanding. We have to identify and distinguish the areas of clustering artificial colors with Hopfield clustering that introduced as Pixel clustering based segmentation method and improve it by Hopfield neural network (HNN) based on spectral properties to show different region by artificial coloring and clustering. We want to improve the technique to use this rule by determining best cluster in neural network.
\end{abstract}

\section{Keywords}

Hopfield, Clustering, CT scan, Brain

\section{INTRODUCTION}

CT scanning - sometimes called CAT scan-a noninvasive procedure that helps doctors to diagnose and treatment of diseases. With the development of X-ray, CT Scan, MRI Image, and other medical imaging techniques. The CT scanner uses digital geometry processing to generate a 3dimensional (3D) image of the inside of an object and Used to Head, Lungs, Cancer, Cardiac, Pulmonary angiogram, Extremities ,.... Like Fig. 1 . weak points of usual method is grayscale images resolution that Scant And makes detection difficult, So we can improve it .. The best way to help Doctor in this kind of analyse is Image processing - we need image segmentation or clustering to analyse CT scan Images also pattern recognition and machine vision techniques.

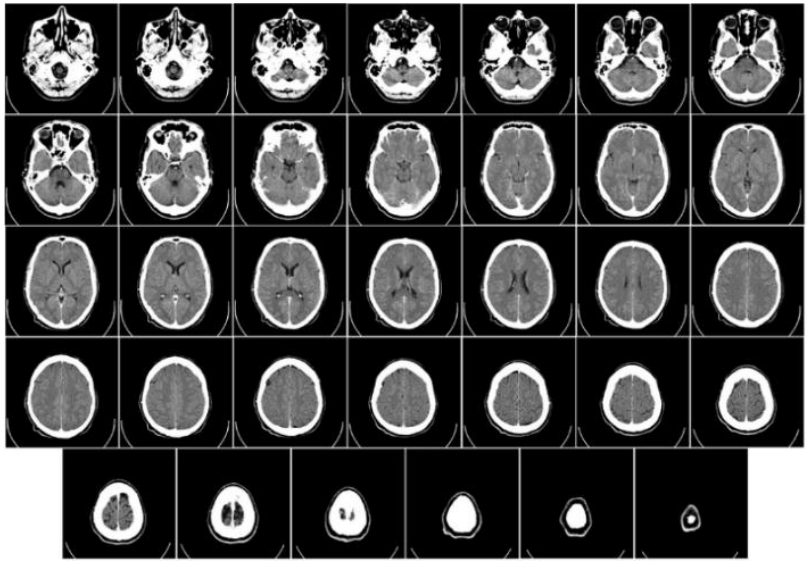

Fig. 1. CT Scan of Brain with multiple Images

In the past method More focus on improving images and coloring or multiple way to assortment pixel . most important way is Region-growing methods, Edge detection, Histogram clustering or classification . segmentation is the process of partitioning an image space into a set of non-overlapping, homogeneous, meaningful regions. The success of any subsequent image analysis depends on the quality of the segmentation (Mitra \& Kundu, 2011 ). In Evaluation has several Phase . Step 1: Determination if it is possible to build CT decision support system, Step 2: Evaluation of the proposed architecture, Step 3: Evaluation of Segmentation quality, Step 4: Evaluation of classification quality based on coloring or clustering. The goal of this paper comprises step 2 and 4 .

\section{PROPOSED METHOD}

The Assortment, which is the first and most important step in any image analysis, especially in this paper clustering is used in order to prepare an image as an input to an automatic vision to extract the outlines of different Areas in the image. In the CT scan image we have a Grayscale picture or create it, So All of the color is Artificial. 


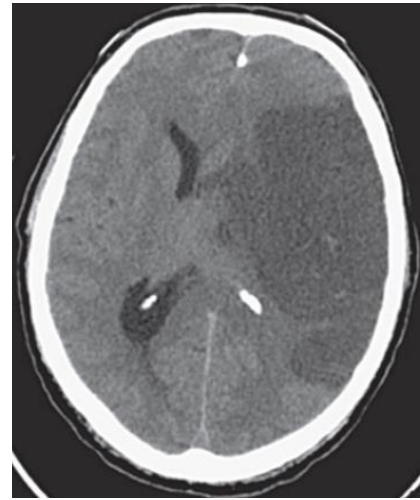

Fig. 2. Apoplexy CT Scan of Brain

The segmentation problem of an image of $\mathrm{N}$ pixels is formulated as a partition of the $\mathrm{N}$ pixels among $\mathrm{M}$ classes such that the assignment of the pixels minimizes a criterion function.

$$
\begin{gathered}
\mathrm{N}=\mathrm{m}^{*} \mathrm{n} \\
\mathrm{X}=\operatorname{reshape}(\operatorname{double}(\mathrm{I}(:,:, 1)),[\mathrm{N}, 1]) \\
\mathrm{u}=\mathrm{kmeans}(\mathrm{X}, \mathrm{K}) \\
\mathrm{U}=\operatorname{zeros}(\mathrm{N}, \mathrm{K}) \\
\text { for } \mathrm{i}=1: \mathrm{N} \\
\mathrm{U}(\mathrm{i}, \mathrm{u}(\mathrm{i}))=\mathrm{u}(\mathrm{i}) \\
\mathrm{V}=\text { zeros }(\mathrm{N}, \mathrm{K}) \\
\mathrm{U} \_ \text {new }=\mathrm{U}
\end{gathered}
$$

Some limitations have been reported due to the network being stuck in an early minimum in local, and this is because the energy landscape in general has more than one minimum in local due to the nonconvex nature of the energy surface. In earlier work (Amartur, Piraino, \& Takefuji, 1992) the authors have made contributions to the algorithm presented by Sammouda et al. (2002) to alleviate this problem by making HNN ( Fig. 3) converge to a local minimum closer to the global minimum in a pre-specified time.

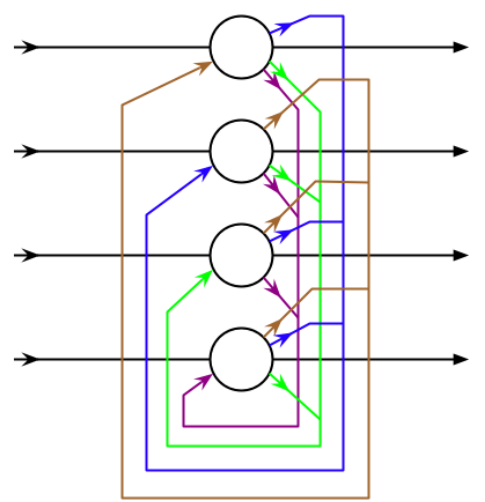

Fig. 3. A Hopfield net with four nodes
Another method is Classification. In general, an automatic image classification method describes an input image (represented as a set of feature vectors). Input of image annotation is a set of feature vectors . Output of automatic image annotation is a subset of dictionary. Hopfield network can be formally described as a complete undirected graph $G=\langle V, f\rangle$, where $V$ is a set and $f: V^{2} \rightarrow R$ is a function that links pairs of nodes to a real value, the connectivity weight .The network is designed to classify the image of $\mathrm{N}$ pixels of $\mathrm{P}$ features among Multiple or $\mathrm{M}$ classes, such that the assignment of the pixels minimizes the following criterion function :

$$
\begin{aligned}
& E= \\
& \frac{1}{2} \sum_{K=1}^{N} \sum_{l=1, k=1}^{M} R_{k l}^{2} V_{k l}^{2}+C(t) \sum_{K=1}^{N} \sum_{l=1, k=1}^{M} N_{k l} V_{k l}(1)
\end{aligned}
$$
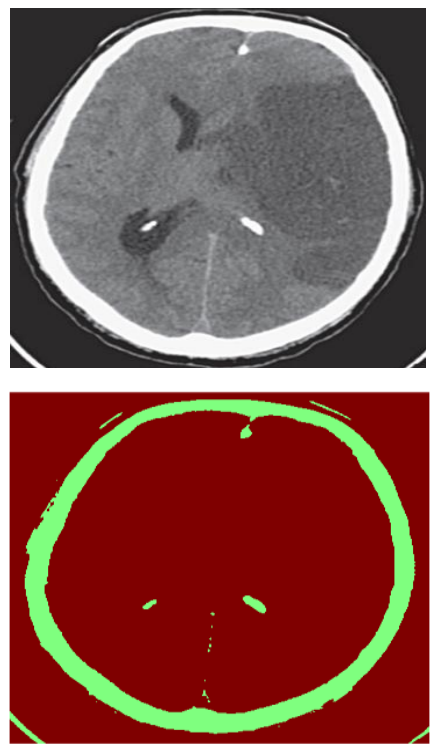

Fig. 4. Segmentation result with two clusters

$x=\left(x_{1}, x_{2}, x_{3}, \ldots \ldots x_{\mathrm{N}}\right)^{\mathrm{T}} \mathrm{Rkl}$ is considered as the Mahalanobis distance (The Mahalanobis distance of an observation from a set of observations with mean

$\mu=\left(\mu_{1}, \mu_{2}, \mu_{3}, \ldots . \mu_{\mathrm{N}}\right)^{\mathrm{T}}$

) measure between the kth pixel and the centroid of class 1 , The connections in a Hopfield net typically have the following restrictions:

$$
\begin{aligned}
& w_{i j}=0, \forall i{ }_{\text {(no unit has a connection with itself) }} \\
& w_{i j}=w_{j i}, \forall i, j j_{\text {(connections are ymmetric) }}
\end{aligned}
$$

and $\mathrm{Vkl}$ is the output of the kth neuron $\mathrm{Nkl}$ is an NXM vector of independent high frequency white noise source .

$$
\begin{aligned}
& \text { Noise }=\operatorname{randi}([0,1],[\mathrm{N}, \mathrm{K}]) \\
& \mathrm{E}=0.5 * \operatorname{sum}(\operatorname{sum}(\mathrm{S}))+\mathrm{c} * \operatorname{sum}(\operatorname{sum}(\text { Noise. } * \mathrm{~V}))
\end{aligned}
$$

$$
E=-\frac{1}{2} \sum_{i, j} \omega_{i j} S_{i} S_{j}+\sum_{i} \theta_{i} S_{i}
$$




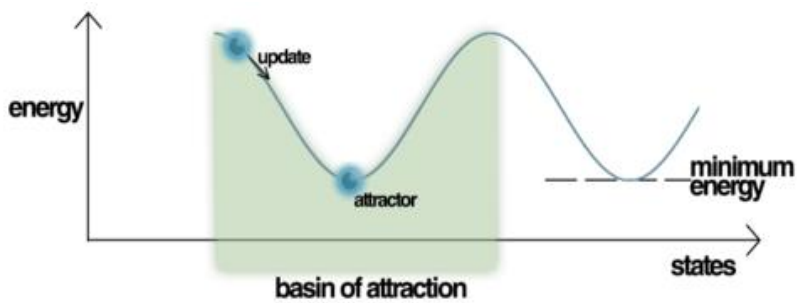

Fig. 5. Energy Landscape of a Hopfield Network

In Fig. 5 see peak and depth that shows the energy of states, its important in Hopfield nueral network and spent energy (formula 4 ). we should be attention number of cluster to have a best peak because Inappropriate cluster has been damaging. Storkey learning rule was introduced by Amos Storkey in 1997 and is both local and incremental. Storkey also showed that a Hopfield network trained using this rule has a greater capacity than a corresponding network trained using the Hebbian rule. The weight matrix of an attractor neural network is said to follow the Storkey learning rule if it obeys:

$$
\begin{aligned}
& \omega_{i j}^{v}=\omega_{i j}^{v-1}+\frac{1}{n} \in_{i}^{v} \in_{j}^{v}-\frac{1}{n} \in_{i}^{v} h_{j i}^{v}- \\
& \frac{1}{n} \epsilon_{i}^{v} h_{i j}^{v}+\varepsilon \\
& h_{i j}^{v}=\sum_{k=1, k \neq i, j}^{n} \omega_{i k}^{v-1} \epsilon_{k}^{v}
\end{aligned}
$$

\section{SEGMANTATION ALGORITHM}

This learning rule is local, since the synapses take into account only neurons at their sides. The rule makes use of more information from the patterns and weights than the generalized, due to the effect of the local field. sector based segmentation algorithm are based on the similarity of the pixels within the Area. The basic formulation involved in sector based segmentation is as follows. The segmentation algorithm For CT Scan Images is :
A. Grayscale images
B. Improve Resolution
C. Initialize the input of the neurons to random values.
D. Apply the following input-output relation.
E. Compute the centroid pixel and the covariance matrix each class 1 as follows
F. Update the inputs of each neuron
G. If $\mathrm{t}<\mathrm{Ts}$, go to Step 2, else terminate

for $\quad t=1$ :Ts- $\quad t<T s \quad U=U \_$new
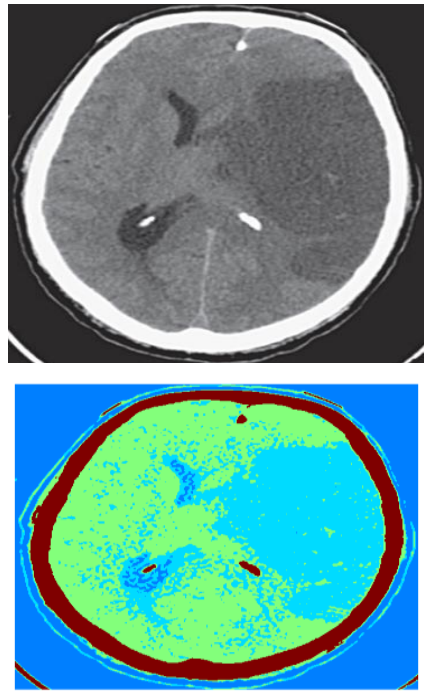

Fig. 6. Segmentation result with four clusters
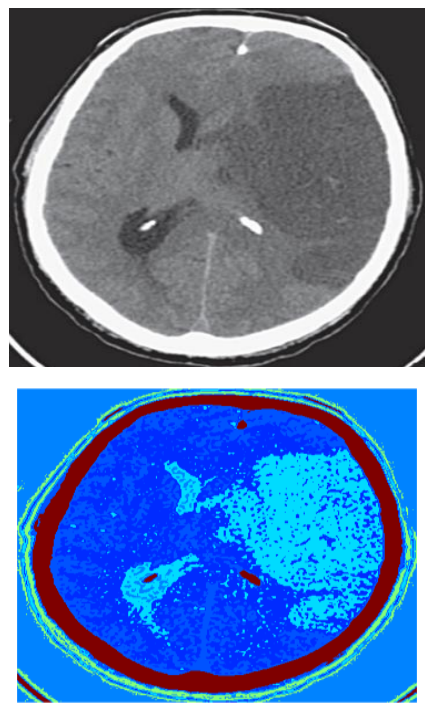

Fig. 7. Segmentation result with six clusters
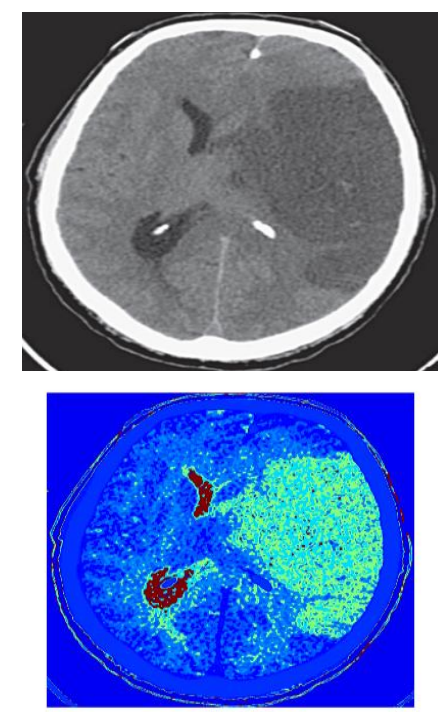

Fig. 8. Segmentation result with Nine clusters 


\section{CONCLUSION}

We accomplishment the (HNN) clustering algorithm to CTScan color images. The segmentation results as generated on different images. We have used different clusters in order to see the accuracy of the algorithm and the sensitivity of the method. For every Unique region and pixel in the input image, HNN returns an index corresponding to a cluster. At the last step this method uses the pixel label to separate object in image based on the color (Blach \& White) and region . with updating any unit by $S_{i} \leftarrow\left\{\begin{array}{c}+1 \text { if } \sum_{j} \omega_{i j} S_{j} \geq \theta_{i} \\ -1 \quad \text { otherwise }\end{array}\right.$ can learn better to network . Fig.Two shows the original CTScan image. The result when applying two clusters HNN segmentation is represented in Fig.Four (To improve) then in Fig.Six Segmentation result with four clusters and in Fig.Seven Segmentation result with six clusters. To means that when we use between four and six cluster our output is real and shown all the aspect . in Fig.eight Segmentation result with Fig.Nine clusters related to Bold colors. We are sorting the Figure in this paper and Indicative that With increase the clustering have a better Output, But if its came very large cluster has an Adverse effects In the future, We can identify the areas Automatically For Doctors And Improve resolution of target region or compare the Changes While treatment to shows At a time. Other one is identifying the kinde and level of a stroke and Disease.

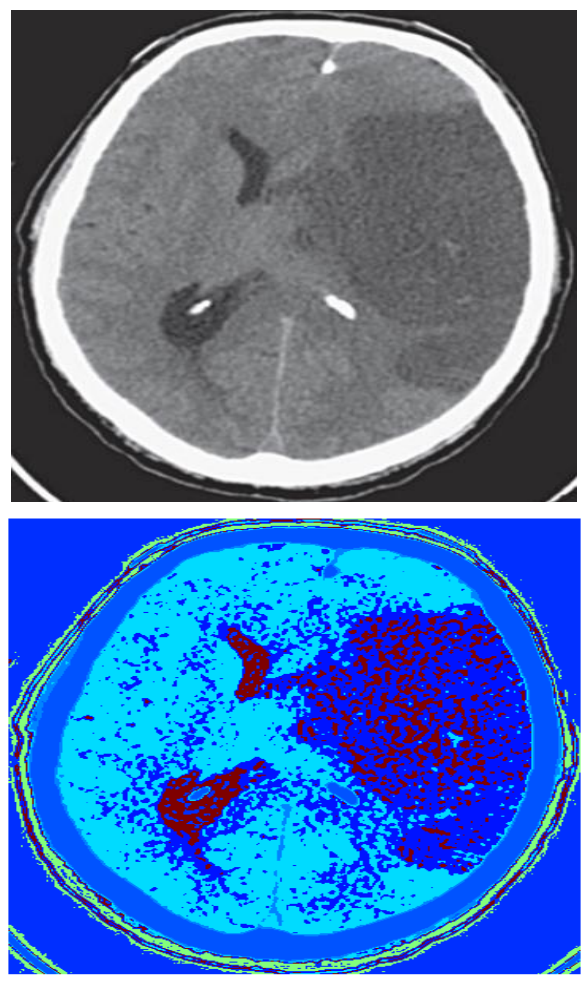

Fig. 9. Segmentation result with Seven clusters

\section{ACKNOWLEDGMENTS}

This Paper is supported by Mr.Hossein Baloochian And Special Thanks to President of Islamic Azad University Of Ferdows in Iran.

\section{REFERENCES}

[1] R.C Gonzalez, R.E Woods and S.L Eddins, Digital Image Processing Using MATLAB, Pears on, Fifth Impres sion,2009.

[2] Coppini G, Diciotti S, Falchini M, Villari N, Valli G. Neural networks for computer-aided diagnosis: detection of lung nodules in chest radiograms. IEEE Transactions on Information Technology in Biomedicine 2003; 34058.

[3] A. Ben-Hur, A. Elisseeff, and I. Guyon, A stability based method for discovering structure in clustered data. In Proc. of Pacific Symposium on Biocomputing, pages 515, 2002.

[4] Pal, S. K., Ghosh, A., \& Uma Shankar, B. (2000). Segmentation of remotely sensed images with fuzzy thresholding, and quantitative evaluation. International Journal of Remote Sensing, 21, 2269-2280.

[5] Sammouda, M., Sammouda, R., Niki, N., \& Mukai, K. (2002). Liver cancer detection system based on the analysis of digitized color images of tissue samples obtained using needle biopsy. Information Visualization, 130-135.

[6] S.K. Pal et al., "A review on Image segmentation techniques', Pattern Recognition, 29, 1277,1294, 1993.

[7] Shi, J., \& Malik, J. (2000). Normalized cuts and image segmentation. IEEE Transactions on Pattern Analysis and Machine Intelligence, 22, 888-902.

[8] Withey DJ, Koles ZJ. Three generations of medical image segmentation: Methods and available software. Int J Bioelectromag. 2007:67-9

[9] Statistical pattern recognition: A review. IEEE Trans PAMI. 2000;4-21.

[10] Cuadra MB, Craene MD, Duay V. Dense deformation field estimation for Atlas-based segmentation of pathological MR brain images. Comput Met Prog Biomed. 2006;84:67-73.

[11] Ge J, Sahiner B, Hadjiiski LM, et al. Computer aided detection of clusters of microcalcifications on full field digital mammograms. Medical Physics 2006;33:2975-87 and Quantitative Cytology and Histology 2007;29(2):101-15.

[12] Pratt KW. Digital image processing. 3rd ed. Willey; 2001. pp. $552-80$

[13] Pal NR, Pal SH. A review on image segmentation techniques. Pattern Recog. 1993;26:1277-91.

[14] Argenti F, Alparone L, Benelli G. Fast algorithm for texture analysis using co-occurrence matrices. IEE Proc Part F: Radar Signal Proc. 1990;137:443-9 